\title{
Pure Point Spectrum Under 1-Parameter Perturbations and Instability of Anderson Localization
}

\author{
A. Ya. Gordon \\ MITPAN, Warshavskoye shosse, 79, korpus 2, Moscow, 113556, Russia
}

Received: 22 March 1993

\begin{abstract}
We consider a selfadjoint operator, $A$, and a selfadjoint rank-one projection, $P$, onto a vector, $\psi$, which is cyclic for $A$. We study the set of all eigenvalues of the operator $A_{t}=A+t P(t \in \mathbb{R})$ that belong to its essential spectrum (which does not depend on the parameter $t$ ). We prove that this set is empty for a dense set of values of $t$. Then we apply this result or its idea to questions of Anderson localization for 1-dimensional Schrödinger operators (discrete and continuous).
\end{abstract}

\section{Introduction}

Let $\left\{A_{t}\right\}_{t \in \mathbb{R}}$ be a one-parameter family of linear selfadjoint operators

$$
A_{t}=A+t P
$$

in a Hilbert space $\mathscr{H}$. Here $A$ is a selfadjoint operator with simple spectrum and $P$ a projection $(\cdot, \psi) \psi$, where $\psi$ is a normed cyclic vector for $A$. All the operators (1) are selfadjoint on $D(A)$ and have the same essential spectrum ${ }^{1}$; denote this closed subset of $\mathbb{R}$ by $\Sigma$.

We will be concerned with the eigenvalues of the operators $A_{t}$ that lie on the essential spectrum, i.e. with the intersection $\sigma_{p}\left(A_{t}\right) \cap \Sigma$ [in the sequel, $\sigma_{p}(B)$, for any linear operator $B$, will denote its point spectrum]. Information about this set can help in studying the nature of the spectrum of $A_{t}$.

In $[\mathrm{SW}]$, a necessary and sufficient condition was found for operators $A_{t}$ to have pure point spectrum for $L$-a.e. $t$. It was formulated in terms of the Stieltjes transform of the spectral measure of $\psi$ for $A$. This criterion was then applied to questions of Anderson localization for random Schrödinger operators. It led, in particular, to the

1 Recall that the essential spectrum $\sigma_{\text {ess }}(B)$ of a selfadjoint operator $B$ (see [Gl]) consists of all nonisolated points of its spectrum and all isolated eigenvalues of infinite multiplicity. The latter case is impossible for operators whose spectrum has finite multiplicity, in particular for the above operators (1) and for one-dimensional difference and differential operators considered in the following 
following statement (see also [DLS]). Let $h(\omega)$ be the random Schrödinger operator of the one-dimensional Anderson model, i.e.

$$
(h(\omega) y)(n)=y(n-1)+y(n+1)+v(n, \omega) y(n) \quad(n \in \mathbb{Z}),
$$

where $\omega \in \Omega[(\Omega, \mathscr{F}, P)$ is a probability space $]$, and values of random potential $v(n, \omega)$ at distinct sites $n$ are independent real random variables with a common distribution $\nu(d x)$. Suppose $\nu$ has a non-trivial absolutely continuous component and $\int\left(\log _{+}|x|\right) \nu(d x)<\infty$. Then with probability 1 , for L-a.e. $t \in \mathbb{R}$ (in particular, for $t=0$ ) the operator $h_{t}(\omega)=h(\omega)+t \delta_{0}$ has only point spectrum. (Here $\delta_{0}$ denotes multiplication by the $\delta$-function supported at 0 .)

A question arises: is the restriction "for $L$-almost every $t$ " in the above result essential? In [SW], an example is indicated where the spectra of operators (1) are pure point for Lebesgue almost all rather than all $t$. However, this example does not provide an answer to a question: what is the situation with Schrödinger operators in $l^{2}(\mathbb{Z})$ ? Is it true that for a "typical" Schrödinger operator $h(\omega)$ (i.e. for any "sufficiently random" stochastic potential, with probability 1) the spectrum of an operator, say, $h(\omega)+t \delta_{0}$ is pure point for all $t$ ? Short of that, is it true for the above-mentioned Anderson model, where the "randomness" of a potential is maximal?

Molchanov raised a related question, which pertained to a one-parameter family of self-adjoint Schrödinger operators $H^{\vartheta}$ in $L^{2}\left(\mathbb{R}_{+}\right)$[see formulas (8) and (9) below]. If a bounded stochastic potential $v=v(x, \omega)$ is ergodic and nondeterministic, then, as it was found by Kotani [Ko], with probability 1 the operator $H^{\vartheta}(\omega)$ has only point spectrum for $L$-a.e. $\vartheta \in[0, \pi)$. Similarly, the spectrum proved to be pure point for some explicit individual potentials (see example [Go] and general result [KMP], which concerns Schrödinger operators in $l^{2}\left(\mathbb{Z}_{+}\right)$), but again under the same restriction: for $L$-a.e. value of the boundary phase. Molchanov raised a question: is this restriction essential? There are known some random potentials for which almost surely (a.s.) the spectrum is pure point for $L$-a.e. rather than all $\vartheta$. However, these potentials are highly symmetrical. Is it true that for a "typical" individual potential the spectrum is pure point for all $\vartheta$ ?

The main result of this paper states that the opposite is true: the restriction "for $L$-almost every $\vartheta$ " (or $t$ ) is quite necessary, at least when the essential spectrum $\Sigma$ has a non-empty interior (which does hold for "typical" potentials). First we establish, for abstract operators (1), the following result.

Theorem 1. There is a thin set $Z$ in $\mathbb{R}$ such that for any $t \in \mathbb{R} \backslash Z$ the intersection $\sigma_{p}\left(A_{t}\right) \cap \Sigma$ is empty.

Then we apply this theorem or its idea to one-dimensional Schrödinger operators and obtain some "negative" results, which show that the localization, though being typical, is however extremely unstable under one-parameter perturbations.

These further results are formulated below. In particular, the answers to the above two questions are contained in Theorems $2^{*}, 2^{* *}$, and 5.

(a) We begin with results concerning operators on $\mathbb{Z}$. Let $\left\{h_{t}\right\}$ be a one-parameter family of linear selfadjoint operators $h_{t}$ in $l^{2}(\mathbb{Z})$ defined by

$$
\left(h_{t} y\right)(n)=y(n-1)+y(n+1)+(v(n)+t \varphi(n)) y(n), \quad n \in \mathbb{Z} .
$$

Here $t \in \mathbb{R} ; v(\cdot)$ ("the potential") and $t \varphi(\cdot)$ ("the perturbation") are real-valued functions on $\mathbb{Z}, \varphi \not \equiv 0$ being non-negative and finitely supported. We denote its support by $S$, so that $1 \leq|S|<\infty$. The operators $h_{t}$ are selfadjoint on the linear 
subspace

$$
\left\{\left.y \in l^{2}(\mathbb{Z})\left|\sum_{n \in \mathbb{Z}} v^{2}(n)\right| y(n)\right|^{2}<\infty\right\} .
$$

Denote the common essential spectrum of all the operators $h_{t}$ by $\Sigma$. Consider the equation $h_{0} y=E y$, or

$$
y(n-1)+y(n+1)+v(n) y(n)=E y(n), \quad n \in \mathbb{Z} .
$$

For some $E$ it may have a non-trivial solution $y(n)$ vanishing on the support of $\varphi:\left.y\right|_{S} \equiv 0$. The set of all such $E$ we denote by $\sigma_{0}$. Evidently, each $E \in \sigma_{0}$ remains an eigenvalue of $h_{t}$ for any $t \in \mathbb{R}$, so that $\sigma_{p}\left(h_{t}\right) \cap \Sigma \supseteq \sigma_{0} \cap \Sigma$ for all $t \in \mathbb{R}$.

Theorem 2. For any $v$ and $\varphi$ ( $v$ real-valued, $\varphi \not \equiv 0$ non-negative and finitely supported) there exists a thin set $Z \subset \mathbb{R}$ such that for any $t \in \mathbb{R} \backslash Z$ the point spectrum $\sigma_{p}\left(h_{t}\right)$ of the operator (3) satisfies

$$
\sigma_{p}\left(h_{t}\right) \cap \Sigma=\sigma_{0} \cap \Sigma .
$$

In other words, for these $t$ only those eigenvalues of $h_{t}$ remain on the essential spectrum which are "immovable" (independent of $t$ ). Note that the set $\sigma_{0}$ of these $t$ is at most finite if $|S| \geq 2$ and is empty if $S$ contains a pair of neighboring points. To be more specific: if $|S| \geq 2$ and $l$ denotes the distance between the two closest sites in $S$, then for all $t \in \mathbb{R} \backslash Z$ we have:

$$
\left|\sigma_{p}\left(h_{t}\right) \cap \Sigma\right|=\left|\sigma_{0} \cap \Sigma\right| \leq l-1 .
$$

In particular, if $|S| \geq 2$ and $\Sigma$ contains a segment, $\Delta$, then for $t \in \mathbb{R} \backslash Z$ the spectrum of $h_{t}$ (contains $\Delta$ and) is purely continuous on $\Delta$, up to a finite number of eigenvalues.

If $|S|=1$, then for an individual potential $v$ nothing prevents $\sigma_{0}$ from being dense in $\Sigma$, even if $\Sigma$ contains a segment, so the above way of proving that the spectrum of $h_{t}$ is not pure point does not work. However for a random ergodic potential some conclusion may be derived in this case, too.

Let

$$
(h(\omega) y)(n)=y(n-1)+y(n+1)+v(n, \omega) y(n) \quad(n \in \mathbb{Z}),
$$

where $v(n, \omega)$ is a stationary ergodic potential, and

$$
h_{t}(\omega)=h(\omega)+t \delta_{0},
$$

where $t \in \mathbb{R}$. According to [P], with probability 1 the spectrum of $h(\omega)$ is a nonrandom closed set coinciding with $\sigma_{\text {ess }}(h(\omega))$; therefore, it contains no isolated points (see the footnoote in the beginning). Denote it by $\Sigma$.

Theorem $2^{*}$. Suppose $\Sigma$ has a non-empty interior int $\Sigma$. Then with probability 1 there exists a thin set $Z(\omega) \subset \mathbb{R}$ such that for any $t \in \mathbb{R} \backslash Z(\omega)$ the spectrum of the operator $h_{t}(\omega)$ is not pure point. Moreover, for these $t, \sigma_{c}\left(h_{t}(\omega)\right)$ contains int $\Sigma$ (the closure of int $\Sigma)$.

Here $\sigma_{c}\left(h_{t}(\omega)\right)$ denotes the spectrum of $\left.h_{t}(\omega)\right|_{\mathscr{H}_{c}}$, where $\mathscr{H}_{c}=\mathscr{H}_{c}(t, \omega)$ is a continuous subspace of $\mathscr{H}$ w.r.t. $h_{t}(\omega)$, that is the subspace

$$
\left\{y \in \mathscr{H} \mid \text { the spectral measure of } y \text { for } h_{t}(\omega) \text { is continuous }\right\} \text {. }
$$

Remark. The essential spectrum of operator (4), for a "random enough" potential $v(n, \omega)$, actually has a non-empty interior. E.g., this is true, if for some periodic 
potential $v_{T}(\cdot) \equiv v_{T}(\cdot+T)$ and arbitrary $\varepsilon>0, L \in \mathbb{N}$, there exists with probability 1 a (random) segment of length $L$ on which $\left|v(\cdot, \omega)-v_{T}(\cdot)\right|<\varepsilon$. (In particular, $v_{T}(\cdot)$ may be a constant.)

Remark. Theorem $2^{*}$ works not only for random enough potentials but even for some deterministic ones, too. In particular, for quasi-periodic potentials $v$ with two basic frequencies which potentials are considered in [CS], the spectrum of $h(\omega)$, as is proved there, is a.s. pure point and has no gaps. Therefore, there must a.s. be a dense set of values of $t$ such that for each of them the spectrum of $h_{t}(\omega)=h(\omega)+t \delta_{0}$ is not pure point.

Theorem $2^{*}$ may be applied, in particular, to the above-mentioned 1-dimensional Anderson model. Actually, in this case we can say more.

Theorem $2^{* *}$. Let

$$
h_{t}(\omega)=h(\omega)+t \varphi,
$$

where $h(\omega)$ is the random operator (2) of the Anderson model and $\varphi$ the operator of multiplication by $\varphi(\cdot)$. Suppose $\varphi(\cdot)$ obeys the same conditions as in Theorem 2 . Then there exists a.s. a thin set $Z(\omega)$ such that for any $t \in \mathbb{R} \backslash Z(\omega)$,

$$
\sigma_{p}\left(h_{t}(\omega)\right) \cap \Sigma=\emptyset .
$$

Remark. For $t \in \mathbb{R} \backslash Z(\omega)$, the spectrum of $h_{t}(\omega)$ consists of the set $\Sigma$ (which is a union of $m$ segments, $1 \leq m \leq \infty)$, where the spectrum is purely continuous, and at most $m \cdot|S|$ isolated eigenvalues.

(b) Define an operator $h^{u}, u \in \mathbb{R}$, in $l^{2}\left(\mathbb{Z}_{+}\right)$by the difference operation $l$,

$$
(l y)(n)=y(n-1)+y(n+1)+v(n) y(n), \quad n \in \mathbb{Z}_{+},
$$

and the boundary condition

$$
y(-1)-u y(0)=0 .
$$

Here $v(\cdot)$ is a real-valued function on $\mathbb{Z}_{+}$. Denote the essential spectrum of the operators $h^{u}$ (it does not depend on $u$ ) again by $\Sigma$.

Theorem 3. For any u, except for some thin set in $\mathbb{R}$, there are no eigenvalues of $h^{u}$ lying on $\Sigma$.

In other words, for such $u, \sigma_{p}\left(h^{u}\right)$ coincides with $\sigma_{\text {discr }}\left(h^{u}\right)$, the set of all isolated points of the spectrum of $h^{u}$.

(c) Let $H_{t}$ denote the operator in $L^{2}(\mathbb{R})$ generated by the differential expression

$$
l y=-y^{\prime \prime}+(v(x)+t \varphi(x)) y, \quad x \in \mathbb{R} .
$$

Here $v(\cdot)$ and $\varphi(\cdot)$ are real-valued, locally integrable functions on $\mathbb{R}, \varphi(\cdot)$ is nonnegative and compactly supported, and mes $\{x: \varphi(x)>0\}>0$. We assume that (7) is of limit-point type at $\pm \infty$ and consequently all the operators $H_{t}$ are selfadjoint (one sufficient condition is: $\int_{-x}^{x} v_{-}(s) d s=0\left(x^{3}\right)$, as $t \rightarrow \infty$ [DS]. Here $v_{-}(s)=$ $\max (-v(s), 0))$.

Theorem 4. For all $t$, except for some thin set in $\mathbb{R}$, there are no eigenvalues of $H_{t}$ on $\Sigma$, the common essential spectrum of all $H_{s}, s \in \mathbb{R}$.

Note that the formulation of Theorem 4 (unlike Theorem 2) does not refer to any set $\sigma_{0}$. It is because of the fact that no eigenfunction of $H_{t}$ can vanish on 
the set $S:=\{x \in \mathbb{R} \mid \varphi(x)>0\}$ having positive measure. But if we admit more general perturbations $\varphi$, namely, non-negative compactly supported measures (e.g., $\left.\varphi(x)=\sum_{k=1}^{m} c_{k} \delta\left(x-x_{k}\right), c_{k}>0\right)$, then in the modified Theorem 4 the set $\sigma_{0}$ appears again, and its formulation becomes similar to that of Theorem 2 .

(d) Consider now a one-parameter family of selfadjoint operators $H^{\vartheta}$ in $L^{2}\left(\mathbb{R}_{+}\right)$ defined by the differential expression

$$
l y=-y^{\prime \prime}+v(x) y \quad(x \geq 0)
$$

and the boundary condition

$$
y(0) \cos \vartheta-y^{\prime}(0) \sin \vartheta=0 \quad(0 \leq \vartheta<\pi) .
$$

Here $v(\cdot)$ is a real-valued function, locally integrable on $[0, \infty)$. For $l$ to be of the limit-point type at $+\infty[C L]$, which is equivalent to the selfadjointness of all the operators $H^{\vartheta}(0 \leq \vartheta<\pi)$, it is sufficient that $\int_{0}^{x} v_{-}(s) d s=O\left(x^{3}\right)$ [DS]. As above, the essential spectra of all the operators $H^{\vartheta}$ coincide with some closed set $\Sigma \subset \mathbb{R}$.

Theorem $5 .^{2}$ Let $v(x), x \geq 0$, be any potential of the limit-point type at $+\infty$. Then for any $\vartheta \in[0, \pi)$, except for some thin subset of $[0, \pi)$, there are no eigenvalues of $H^{\vartheta}$ lying on its essential spectrum $\Sigma$.

The paper is organized as follows. In Sect. 2, Theorem 1 is split into two propositions and the first of them is proved. In Sect. 3, Proposition 2 is established, which completes the proof of Theorem 1. In Sect. 4, "determinate" Theorems 2 and 3 are proved and the modifications are indicated that enable one to prove Theorems 4 and 5. In Sect. 5, we prove "random" Theorems $2^{*}$ and $2^{* *}$.

\section{Proof of Theorem 1 (beginning)}

Theorem 1 is readily deduced from the following two statements. Before formulating them we define some subset of the spectral axis.

Let $D$ be the set of all eigenvalues of all the operators $A_{t}$ :

$$
D:=\bigcup_{t \in \mathbb{R}} \sigma_{p}\left(A_{t}\right)
$$

Proposition 1. $D \cap \Sigma$ is a thin set in $\mathbb{R}$.

Proposition 2. Let $Y$ be a thin set in $\mathbb{R}$. Then

$$
\left(t \in \mathbb{R} \mid \sigma_{p}\left(A_{t}\right) \cap Y \neq \emptyset\right\}
$$

is a thin set, too.

In order to deduce Theorem 1 from these two propositions, it suffices to set $Y:=D \cap \Sigma$ and note that

$$
\sigma_{p}\left(A_{t}\right) \cap Y=\sigma_{p}\left(A_{t}\right) \cap \Sigma \quad \text { for all } t \in \mathbb{R} .
$$

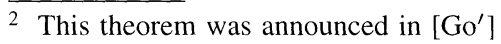


Proof of Proposition 1. Define a set

$$
\begin{aligned}
Q:= & \left\{\xi=(t, E) \in \mathbb{R}^{2} \mid \text { there exists a solution } y \neq 0\right. \\
& \text { to the equation } \\
& \left.A_{t} y=E y\right\} .
\end{aligned}
$$

We will denote by $p$ and $\pi$ the orthogonal projections of the plane

$$
\mathbb{R}^{2}=\{\xi=(t, E) \mid t, E \in \mathbb{R}\}
$$

onto the $E$ - and $t$-axis respectively. Obviously,

$$
D=p(Q) \text {. }
$$

Lemma 1. For any non-trivial solution y of Eq. (10) we have

$$
(y, \psi) \neq 0 \text {. }
$$

Proof. Assume that $(y, \psi)=0$. Then $P y=0$ and hence $A y=E y$; it follows that for any $\mu \in \mathbb{C} \backslash \mathbb{R}$,

$$
\left((A-\mu)^{-1} y, \psi\right)=\left((E-\mu)^{-1} y, \psi\right)=0,
$$

so that $\left(y,(A-\bar{\mu})^{-1} \psi\right)=0$. Since $\psi$ is cyclic for $A$, the closed linear span of the set

$$
\left\{(A-\lambda)^{-1} \psi \mid \lambda \in \mathbb{C} \backslash \mathbb{R}\right\}
$$

is dense in $\mathscr{H}$. Thus $y=0$.

Corollary. The linear space of all solutions to (10) is at most one-dimensional.

Next we define, for $M>0$, one more set in $\mathbb{R}^{2}$ :

$$
\begin{aligned}
Q_{M}:= & \left\{\xi=(t, E) \in \mathbb{R}^{2} \mid \text { there exists a solution } y\right. \text { to Eq. (10) such that } \\
& (y, \psi)=1 \\
& \text { and } \\
& (y, y) \leq M\} .
\end{aligned}
$$

Lemma 2. The set $Q_{M}$ is closed.

Proof. Let $Q_{M} \ni \xi_{n}=\left(t_{n}, E_{n}\right) \rightarrow \xi=(t, E) \in \mathbb{R}^{2}(n \rightarrow \infty)$. Denote the corresponding solutions to (10) satisfying (11) and (12) by $y_{n}$. The sequence $\left\{y_{n}\right\}_{n=1}^{\infty}$ is bounded in $\mathscr{H}$, and by the weak sequential compactness of closed balls in Hilbert space we may assume that $y_{n}$ converges weakly to some vector $y \in \mathscr{H}$. Evidently, $y$ satisfies relations (11) and (12). Moreover, $y$ satisfies (10). Indeed, as far as

$$
A y_{n}+t_{n}\left(y_{n}, \psi\right) \psi=E_{n} y_{n}
$$

and $y_{n} \stackrel{w}{\rightarrow} y$ (in such a way the weak convergence in $\mathscr{H}$ is denoted), we have: $A y_{n} \stackrel{w}{\rightarrow} z$, where

$$
z+t(y, \psi) \psi=E y
$$

The selfadjoint operator $A$ is closed, i.e. its graph is a closed and hence a weakly closed linear subspace of $\mathscr{H} \oplus \mathscr{H}$. Consequently, $y$ belongs to the domain of $A$ and $z=A y$. Therefore, (13) implies (10). And it follows from (10), (11), and (12) that $\xi=(t, E) \in Q_{M}$. 
Further, let $T$ be any positive number; we define two more sets $Q_{M}^{T} \in \mathbb{R}^{2}$, $D_{M}^{T} \in \mathbb{R}$, setting

$$
Q_{M}^{T}:=Q_{M} \cap \pi^{-1}([-T, T])
$$

and

$$
D_{M}^{T}:=p\left(Q_{M}^{T}\right) .
$$

Both sets are closed (regarding the former it is obvious, while the latter is closed because for any segment $\Delta \subset \mathbb{R}$ the set

$$
D_{M}^{T} \cap \Delta=p\left(Q_{M}^{T} \cap p^{-1}(\Delta)\right)
$$

is an $E$-projection of a compact set and, therefore, is compact itself).

As far as

$$
D=\bigcup_{M=1}^{\infty} \bigcup_{T=1}^{\infty} D_{M}^{T},
$$

Proposition 1 is a consequence of the following statement.

For any positive $M$ and $T$, the closed set $D_{M}^{T} \cap \Sigma$ is nowhere dense in $\mathbb{R}$.

In other words, the set

$$
\operatorname{int}\left(D_{M}^{T} \cap \Sigma\right)=\left(\operatorname{int} D_{M}^{T}\right) \cap(\operatorname{int} \Sigma)
$$

is empty. One can say more. The following assertion is true.

Proposition $1^{*}$. The set (int $\left.D_{M}^{T}\right) \cap \Sigma$ is empty.

The proof exploits the following three lemmas.

Lemma 3. Let $\xi=(t, E), \xi^{\prime}=\left(t^{\prime}, E^{\prime}\right)$ belong to $Q$ and $y, y^{\prime}$ be solutions to the corresponding equations (10) any $A_{t^{\prime}} y^{\prime}=E^{\prime} y^{\prime}$, normalized by conditions (11) and $\left(y^{\prime}, \psi\right)=1$ respectively. Then

$$
t^{\prime}-t=\left(E^{\prime}-E\right)\left(y^{\prime}, y\right) \text {. }
$$

It follows from Lemma 3 that the set $Q \subset \mathbb{R} \times D$ is a graph (up to a permutation of axes) of some map $\tau: D \rightarrow \mathbb{R}:$

$$
Q=\left\{\xi=(t, E) \in \mathbb{R}^{2} \mid E \in D, t=\tau(E)\right\} .
$$

Lemma 4. The restriction $\left.\tau\right|_{D_{M}^{T}}$ satisfies Lipschitz condition (and, in particular, is continuous):

$$
\left|\tau\left(E_{1}\right)-\tau\left(E_{2}\right)\right| \leq M\left|E_{1}-E_{2}\right| .
$$

Proof. Make use of (14), (12), and the Schwarz inequality.

For $\xi=(t, E) \in Q$, we will denote the unique vector $y$, satisfying (10) and (11), by $y^{\xi}$ (the uniqueness is ensured by Corollary to Lemma 1 ).

Lemma 5. As $Q_{M} \ni \xi^{\prime} \rightarrow \xi\left(\in Q_{M}\right)$, we have

$$
y^{\xi^{\prime}} \stackrel{w}{\rightarrow} y^{\xi}
$$

Proof. In the converse case, there exist a vector $z \in \mathscr{H}$ and a sequence $\xi_{n} \rightarrow \xi$ $\left(\xi_{n} \in Q_{M}, \xi \in Q_{M}\right)$ so that for all $n=1,2, \ldots$,

$$
\left|\left(y^{\xi_{n}}, z\right)-\left(y^{\xi}, z\right)\right| \geq \varepsilon,
$$


where $\varepsilon$ is a positive constant. In view of the weak sequential compactness of closed balls in Hilbert space, the sequence $\left\{y^{\xi_{n}}\right\}$ (after some "thinning out," if necessary) may be thought of as weakly convergent to some vector $y$. This vector satisfies relations (11), (12), and (10). By Corollary to Lemma 1,

$$
y=C y^{\xi}
$$

and since $(y, \psi)=\left(y^{\xi}, \psi\right)=1$, it follows that $y=y^{\xi}$. Therefore, $y^{\xi_{n}} \stackrel{w}{\rightarrow} y^{\xi}$. But this contradicts (15).

Since distinct points $\xi=(t, E)$ from $Q$ have distinct $E$-coordinates (Lemma 3), an alternative notation $y^{E}$ for $y^{\xi}$ is also possible.

Lemma 6. As $D_{M}^{T} \ni E^{\prime} \rightarrow E\left(\in D_{M}^{T}\right)$, we have

$$
\frac{\tau\left(E^{\prime}\right)-\tau(E)}{E^{\prime}-E} \rightarrow\left\|y^{E}\right\|^{2}
$$

Proof. This assertion is a mere consequence of Lemmas 3, 4, and 5.

Proof of Proposition $1^{*}$. Assume the converse. Then there exists some point $E_{0} \in$ (int $\left.D_{M}^{T}\right) \cap \Sigma$. Let $\varepsilon>0$ be so small that the segment

$$
\Delta=\left[E_{0}-\varepsilon, E_{0}+\varepsilon\right]=:\left[E_{1}, E_{2}\right]
$$

is contained in the open set int $D_{M}^{T}$. Then for any $E \in \Delta$ there exist some $t \in[-T, T]$ [namely, $t=\tau(E)$ ] and solution $y^{E}$ to Eq. (10) such that relations (11) and (12) are satisfied.

For $E \in \Delta$ we will write $t^{E}$ instead of $\tau(E)$. The function $E \mapsto t^{E}: \Delta \rightarrow \mathbb{R}$, according to Lemma 6 , is differentiable and satisfies the relation

$$
\frac{d t^{E}}{d E}=\left\|y^{E}\right\|^{2}>0 .
$$

Let $t \mapsto E^{t}$ be an inverse function. It maps the segment $\left[t_{1}, t_{2}\right]$ (where $t_{j}=t^{E_{\jmath}}$, $j=1,2)$ onto the segment $\Delta=\left[E_{1}, E_{2}\right]$.

Let $E \in \Delta$ and $t=t^{E}$. By (10), $y^{E}$ is an eigenvector of the operator $A_{t}=A+t P$, and the corresponding eigenvalue is $E$. According to the Corollary to Lemma 1 , this eigenvalue is simple. by

Denote by $\mu_{t}$ the spectral measure of the vector $\psi$ for $A_{t}$. This measure is defined

$$
\mu_{t}(X)=\left(\mathscr{E}_{t}(X) \psi, \psi\right)
$$

where $\mathscr{E}_{t}(\cdot)$ is the resolution of the identity corresponding to $A_{t}$, and $X$ is an arbitrary Borelian subset of $\mathbb{R}$. Since $\mathscr{C}_{t}(\{E\})$ is the orthogonal projection onto the vector $y^{E} /\left\|y^{E}\right\|$, the spectral measure $\mu_{t}$ has at the point $E=E_{t}$ an atom, whose mass is

$$
\begin{aligned}
\alpha(E):=\mu_{t}(\{E\}) & =\left(\psi, \frac{y^{E}}{\left\|y^{E}\right\|}\right)^{2}=\frac{1}{\left\|y^{E}\right\|^{2}} \\
& =\left(\frac{d t^{E}}{d E}\right)^{-1}=\frac{d E^{t}}{d t}
\end{aligned}
$$


[we make use of (16)]. Therefore,

$$
\begin{aligned}
\int_{-\infty}^{\infty} \mu_{t}(\Delta) d t & \geq \int_{t_{1}}^{t_{2}} \mu_{t}(\Delta) d t \geq \int_{t_{1}}^{t_{2}} \alpha\left(E^{t}\right) d t \\
& =\int_{t_{1}}^{t_{2}} \frac{d E^{t}}{d t} d t=E_{2}-E_{1}=|\Delta|
\end{aligned}
$$

Now we need the following statement.

Lemma 8 (see [DLS]).

$$
\int_{-\infty}^{\infty} \mu_{t}(\Delta) d t=|\Delta| .
$$

(We need only the inequality " $\leq$ "; this fact is essentially proved in [DLS]. Formally, the proof in [DLS] refers to one- and quasi-one-dimensional Schrödinger operators, but actually it is suited to the general case as well).

By Lemma 8, both inequalities in (18) actually turn into equalities. This means that for $L$-a.e. $t \in \mathbb{R}$ the restriction of the measure $\mu_{t}$ to the segment $\Delta$ either reduces to one atom at the point $E^{t}$ (when $t_{1} \leq t \leq t_{2}$ ) or is the zero measure. In both cases the essential spectrum of the operator $A_{t}$ contains no inner points of $\Delta$.

On the other hand, the essential spectrum of $A_{t}$ is independent of $t$ and coincides with $\Sigma$ for all $t$; therefore, we have

$$
\text { (int } \Delta) \cap \Sigma \equiv\left(E_{0}-\varepsilon, E_{0}+\varepsilon\right) \cap \Sigma=\emptyset \text {. }
$$

In particular, $E_{0} \notin \Sigma$, which contradicts the assumption.

Proposition $1^{*}$ is proved, and, along with it, Proposition 1 is proved, too.

\section{Proof of Proposition 2}

Let $Y$ be a thin subset of the $E$-axis. We are concerned with the set

$$
X:=\left\{t \in \mathbb{R} \mid \sigma_{p}\left(A_{t}\right) \cap Y \neq \emptyset\right\} .
$$

Since for any fixed $t$ the set $\{E \in \mathbb{R} \mid(t, E) \in Q\}$ coincides with $\sigma_{p}\left(A_{t}\right)$, the set in question, $X$, can be obtained as follows:

$$
X=\pi\left(Q \cap p^{-1}(Y)\right) .
$$

Our aim is to prove that $X$ is a thin set if such is $Y$. Let $\left\{Y_{n}\right\}$ be an increasing sequence of closed nowhere dense subsets of $\mathbb{R}$ whose union contains $Y$. Further, $Q$ is a union of an increasing sequence of compact sets, e.g. the sets

$$
Q^{(M)}:=Q_{M} \cap p^{-1}([-M, M]) \cap \pi^{-1}([-M, M]), \quad M=1,2, \ldots .
$$

Hence it is enough to establish the following statement.

Proposition 2*. Suppose $K$ is a compact subset of $Q_{M}$ and its E-projection, $p(K)$, is nowhere dense. Then its t-projection, $\pi(K)$, is nowhere dense as well. 
(Applying this statement to the compact sets $K_{M}=Q^{(M)} \cap p^{-1}\left(Y_{M}\right)(M=$ $1,2, \ldots)$, we shall obtain, in view of (19): $X \subseteq \bigcup_{M=1}^{\infty} \pi\left(K_{M}\right)$, the summands $\pi\left(K_{M}\right)$ being nowhere dense. Therefore, it will be proved that $X$ is thin).

Proof of Proposition 2*. Assume that, on the contrary, there exists a compact set $K \subseteq Q_{M}$ such that its $E$-projection, $p(K)$, is nowhere dense but its $t$-projection, $\pi(K)$, contains a non-trivial segment, $I$.

Consider the set, $W$, of all compact sets $K^{\prime}\left(K^{\prime} \subseteq K\right)$, for which $\pi\left(K^{\prime}\right) \supseteq I$. Put $W$ in partial order by inclusion. Let $Z$ be an arbitrary chain in $W$; set

$$
\tilde{K}:=\bigcap_{K^{\prime} \in Z} K^{\prime}
$$

It is easily seen that $\tilde{K} \in W$. Therefore, any chain in $W$ is bounded from below. By Zorn's lemma, there exists at least one minimal element, say $K_{0}$, in $W$. We may assume that $K=K_{0}$, i.e. assume from the very beginning that the compact set $K$ is minimal: if $K^{\prime} \subset K, K^{\prime} \neq K, K^{\prime}$ compact, then $\pi\left(K^{\prime}\right)$ does not contain the segment I. (In fact, obviously, $\pi\left(K^{\prime}\right) \varsubsetneqq \pi(K)=I$ ).

Consider the map $\xi \mapsto y^{\xi}: K \rightarrow \mathscr{H}$, which associates to any $\xi=(t, E) \in K$ the above solution $y^{\xi}$ of Eq. (10). Since $K \subseteq Q_{M}$, by Lemma 5 we have

$$
y^{\xi^{\prime}} \stackrel{w}{\rightarrow} y^{\xi} \quad \text { as } \quad K \ni \xi^{\prime} \rightarrow \xi(\in K) .
$$

Let $\left\{e_{n}\right\}_{n=1}^{\infty}$ be an orthonormal basis in $\mathscr{H}$. Consider a sequence of functions on $K$ :

$$
J_{m}(\xi):=\sum_{n=1}^{m}\left(y^{\xi}, e_{n}\right)^{2}, \quad(\xi \in K, m=1,2, \ldots) .
$$

As $m \rightarrow \infty, J_{m}(\xi)$ converges pointwise to $J_{K}(\xi)$, the restriction of the function $\xi \mapsto\left\|y^{\xi}\right\|^{2}: Q \rightarrow \mathbb{R}$ to $K$. It follows from (20) that each of the functions $J_{m}(\xi)$ is continuous on $K$. By virtue of Baire's theorem (see [O]), the limit function, $J_{K}(\xi)$, is continuous at some point $\xi_{c} \in K$.

Lemma 9. As $\xi, \xi^{\prime}$ tend to $\xi_{c}\left(\xi, \xi^{\prime} \in K ; \xi \neq \xi^{\prime}\right)$,

$$
\frac{t-t^{\prime}}{E-E^{\prime}} \rightarrow J_{K}\left(\xi_{c}\right)
$$

Proof. By Lemma 3,

$$
\frac{t-t^{\prime}}{E-E^{\prime}}=\left(y^{\xi}, y^{\xi^{\prime}}\right)
$$

As $K \ni \xi \rightarrow \xi_{c}$, we have: $y^{\xi \stackrel{w}{\rightarrow}} y^{\xi_{c}}$ and (due to the choice of $\xi_{c}$ ) $\left\|y^{\xi}\right\| \rightarrow\left\|y^{\xi_{c}}\right\|$. These two relations are well-known to imply the strong convergence $y^{\xi} \rightarrow y^{\xi_{c}}$. [Proof: $\left(y^{\xi}-y^{\xi_{c}}, y^{\xi}-y^{\xi_{c}}\right)=\left\|y^{\xi}\right\|^{2}-\left(y^{\xi}, y^{\xi_{c}}\right)-\left(y^{\xi_{c}}, y^{\xi}\right)+\left\|y^{\xi_{c}}\right\|^{2} \rightarrow 0$.] Similarly, $y^{\xi^{\prime}} \rightarrow y^{\xi_{c}}$. Hence, in view of the continuity of the scalar product in $\mathscr{H}$, we obtain:

$$
\frac{t-t^{\prime}}{E-E^{\prime}}=\left(y^{\xi}, y^{\xi^{\prime}}\right) \rightarrow\left\|y^{\xi_{c}}\right\|^{2}
$$

It follows from Lemma 9 and positivity of the function $J_{K}(\cdot)$ that if $U$ is a small enough neighborhood of the point $\xi_{c}$ in $\mathbb{R}^{2}$, then for any $\xi, \xi^{\prime} \in K_{U}:=K \cap \bar{U}$ $\left(\xi \neq \xi^{\prime} ; \bar{U}\right.$ denotes the closure of $U$ ) we have not only $E \neq E^{\prime}$ but also $t \neq t^{\prime}$. So, 
both projections of the compact set $K_{U}, p\left(K_{U)}\right.$ and $\pi\left(K_{U}\right)$, are homeomorphic to it and, consequently, to each other.

By the assumption, $p\left(K_{U}\right)$, being a subset of $p(K)$, contains no non-trivial segments or, which is the same, no homeomorphic images of the segment $[0,1]$; hence, $\pi\left(K_{U}\right)$ contains no non-trivial segments as well. Therefore, $\pi(K \cap U)$, being its subset, is nowhere dense in $I$; now it follows from inclusions $\pi(K \cap U) \cup \pi(K \backslash U) \supseteq \pi(K) \supseteq I$ that the set $\pi(K \backslash U)$ is dense in $I$ and, being compact, contains $I$.

On the other hand, the compact set $K \backslash U$ is a proper subset of $K$ (it does not contain $\xi_{c}$ ). This contradicts the assumed minimality of $K$. Proposition $2^{*}$ and thereby Proposition 2 are proved, so the proof of Theorem 1 is complete.

\section{Proofs of "Determinate" Theorems}

Proof of Theorem 3. Here $\mathscr{H}=l^{2}\left(\mathbb{Z}_{+}\right)$and the operator $h^{u}$ is generated by the difference operation $l$,

$$
(l y)(n)=y(n-1)+y(n+1)+v(n) y(n) \quad(n \geq 0)
$$

and the boundary condition

$$
y(-1)=u y(0) .
$$

Denote by $e_{j}(j=0,1,2, \ldots)$ the vector of the canonical basis associated to the site $j \in \mathbb{Z}_{+}$. It is easy to see that

$$
h^{u}=h+u P \text {, }
$$

where $h=h^{0}$ corresponds to the Dirichlet boundary condition $y(-1)=0$ and $P$ is the orthogonal projection $y \mapsto\left(y, e_{0}\right) e_{0}$. Since

$$
h^{k} e_{0}=\sum_{j=0}^{k-1} c_{j}^{k}(v) e_{j}+e_{k} \quad(k=1,2, \ldots),
$$

where $c_{j}^{k}(v)(j=0,1, \ldots, k-1)$ are polynomials in $v(0), v(1), \ldots, v(k-1)$, it follows that $e_{0}$ is a cyclic vector for $h$, and hence Theorem 3 is a mere consequence of Theorem 1.

Proof of Theorem 2 does not reduce to a direct application of Theorem 1. However, the proof is partly parallel to that of Theorem 1 and exploits one of its intermediate results.

Denote by $D^{*}$ the set of all eigenvalues of all the operators $h_{t}$ :

$$
D^{*}:=\bigcup_{t \in \mathbb{R}} \sigma_{p}\left(h_{t}\right)
$$

Theorem 2 is a consequence of the following two propositions.

Proposition 3. $D^{*} \cap \Sigma$ is a thin set in $\mathbb{R}$.

Proposition 4. Let $Y$ be a thin set in $\mathbb{R}$. Then

$$
X:=\left\{t \in \mathbb{R} \mid\left(\sigma_{p}\left(h_{t}\right) \backslash \sigma_{0}\right) \cap Y \neq \emptyset\right\}
$$

is a thin set, too. 
To deduce Theorem 2 from these two propositions, it is enough to set $Y:=D^{*} \cap \Sigma$ and note that

$$
\left(\sigma_{p}\left(h_{t}\right) \backslash \sigma_{0}\right) \cap Y=\left(\sigma_{p}\left(h_{t}\right) \backslash \sigma_{0}\right) \cap \Sigma .
$$

Proof of Proposition 3. Let $a$ and $b(a \leq b)$ be any entire numbers such that $[a, b] \cap \mathbb{Z} \supseteq S$. In the Hilbert space, $l^{2}(b+1, \infty)$, of all the square-summable sequences $\{y(n)\}_{n=b+1}^{\infty}$ with the usual scalar product we define an operator, $h_{+}$, generated by the difference operation $l$,

$$
(l y)(n)=y(n-1)+y(n+1)+v(n) y(n), \quad n \geq b+1
$$

and the Dirichlet boundary condition

$$
y(b)=0 .
$$

In a similar way we define an operator $h_{-}$in $l^{2}(-\infty, a-1)$. The essential spectra of the operators $h_{+}$and $h_{-}$we denote by $\Sigma_{+}$and $\Sigma_{-}$respectively. According to [Gl], a real number $E$ belongs to $\Sigma_{+}$, if and only if there exists a sequence of finitely supported functions $y_{k}(\cdot) \in l^{2}(b+1, \infty)(k=1,2, \ldots)$ such that their supports go to $+\infty$ (say, $y_{k}(n)=0$ if $n \leq k$ ), and

$$
\left\|l y_{k}-E y_{k}\right\|<\varepsilon_{k}\left\|y_{k}\right\|,
$$

where $\varepsilon_{k} \rightarrow 0(k \rightarrow \infty)$. Similar criteria are valid for $\Sigma_{+}$and $\Sigma$ ( $\Sigma$ was defind above as the common essential spectrum of all the operators $h_{t}, t \in \mathbb{R}$. In this case the finitely supported function $y_{k}(\cdot) \in l^{2}(\mathbb{Z})$ must satisfy (22) and vanish on the "segment" $[-k, k] \cap \mathbb{Z})$. It follows that $\Sigma=\Sigma_{+} \cup \Sigma_{-}$; since the union of any two thin sets is thin, Proposition 3 will be ascertained, if we prove the following statement.

The intersection $D^{*} \cap \Sigma_{+}$is thin.

To prove this, we consider two families of operators, $\left\{h_{1}^{u}\right\}_{u \in \mathbb{R}}$ and $\left\{h_{2}^{u}\right\}_{u \in \mathbb{R}}$. The operator $h_{1}^{u}$ acts in $l^{2}(b+1, \infty)$ and is generated by the difference operation (21) and the boundary condition $y(b)=u y(b+1)$. The operator $h_{2}^{u}$ acts in $l^{2}(b+2, \infty)$ and is generated by the same difference operation $l$ (for $n \geq b+2$ ) and the boundary condition $y(b+1)=u y(b+2)$.

According to the above characterization of the essential spectrum, the essential spectra of all these operators are the same and coincide with $\Sigma_{+}$. Define the sets

$$
D_{1}:=\bigcup_{u \in \mathbb{R}} \sigma_{p}\left(h_{1}^{u}\right)
$$

and

$$
D_{2}:=\bigcup_{u \in \mathbb{R}} \sigma_{p}\left(h_{2}^{u}\right) .
$$

Applying Proposition 1 to both families of operators, we obtain: $D_{1} \cap \Sigma_{+}$and $D_{2} \cap \Sigma_{+}$ are thin sets. Since for any non-trivial solution $y$ to the equation $h_{t} y=E y$, or

$$
y(n-1)+y(n+1)+(v(n)+t \varphi(n)) y(n)=E y(n), \quad(n \in \mathbb{Z}),
$$

we have either $y(b+1) \neq 0$ or $y(b+2) \neq 0$, it follows that $D^{*} \subseteq D_{1} \cup D_{2}$. Hence, $D^{*} \cap \Sigma_{+} \subseteq\left(D_{1} \cap \Sigma_{+}\right) \cup\left(D_{2} \cap \Sigma_{+}\right)$and, therefore, $D^{*} \cap \Sigma_{+}$is thin. Proposition 3 is proved.

Proof of Proposition 4 is similar to that of Proposition 2 but differs in some details. The proof exploits an obvious lemma, analogous to Lemma 3. 
Lemma 10. Let $y(\cdot), \tilde{y}(\cdot)$ belong to $l^{2}(\mathbb{Z})$ and satisfy the following equations:

$$
\begin{aligned}
& h_{t} y=E y, \\
& h_{\tilde{t}} \tilde{y}=\tilde{E} \tilde{y} .
\end{aligned}
$$

Then

$$
(\tilde{t}-t)(\tilde{y}, y)_{\varphi}=(\tilde{E}-E)(\tilde{y}, y) .
$$

Here we denote by $(\cdot, \cdot)$ the scalar product in $l^{2}(\mathbb{Z})$ and by $(\cdot, \cdot)_{\varphi}$ a quantity

$$
(y, z)_{\varphi}=\sum_{n \in S} \varphi(n) y(n) \overline{z(n)} .
$$

We must prove that the set

$$
X=\left\{t \in \mathbb{R} \mid\left(\sigma_{p}\left(h_{t}\right) \backslash \sigma_{0}\right) \cap Y \neq \emptyset\right\}
$$

is thin if such is $Y$. Define the set

$$
\begin{aligned}
Q:= & \left\{\xi=(t, E) \in \mathbb{R}^{2} \mid \text { there exists a solution } y(\cdot)\right. \\
& \text { to Eq. (23) such that } \left.\left.y\right|_{S} \neq \equiv\right\} .
\end{aligned}
$$

Since for any fixed $t$ the set $\{E \in \mathbb{R} \mid(t, E) \in Q\}$ coincides with $\sigma_{p}\left(h_{t}\right) \backslash \sigma_{0}$, the set $X$ can be written in the form:

$$
X=\pi\left(Q \cap p^{-1}(Y)\right] .
$$

For $M>0$ we define a subset $Q_{M}$ of $Q$ by

$$
\begin{aligned}
Q_{M}:= & \left\{\xi=(t, E) \in \mathbb{R}^{2} \mid\right. \text { there exists a solution } \\
& y(\cdot) \text { to }(23) \text { satisfying } \\
& (y, y)_{\varphi}=1 \\
& \text { and } \\
& (y, y) \leq M\}
\end{aligned}
$$

It follows from the weak sequential compactness of closed balls in the Hilbert space and the finiteness of $S=\operatorname{supp} \varphi$ that $Q_{M}$ is closed in $\mathbb{R}^{2}$. To prove Proposition 4 , it is enough to establish the following statement.

Proposition $4^{*}$. Let $K$ be a compact subset of $Q_{M}$ and $p(K)$ be nowhere dense. Then $\pi(K)$ is nowhere dense as well.

Proof. Let us assume the converse: there exists a compact set $K \subseteq Q_{M}$ such that its $E$-projection, $p(K)$, is nowhere dense but its $t$-projection, $\pi(K)$, contains a nontrivial segment, $I$.

Since $K \subseteq Q_{M}$, for any $\xi=(t, E) \in K$ there exists a solution $y^{\xi}(\cdot)$ to (23) satisfying relations (25) and (26). Such a solution, if chosen to be real-valued, is unique up to multiplication by \pm 1 (the standard Wronskyan argument). Suppose that such a selection of $y^{\xi}(\cdot)$ is made for each $\xi \in K$.

Lemma 11. There exists a map $\left(\xi^{\prime}, \xi\right) \mapsto c\left(\xi^{\prime}, \xi\right): K \times K \rightarrow\{+1,-1\}$ such that for any $\xi_{0} \in K$,

$$
c\left(\xi, \xi_{0}\right) y^{\xi \stackrel{w}{\rightarrow}} y^{\xi_{0}} \quad \text { in } \quad l^{2}(\mathbb{Z})
$$

and

$$
\left.\left.c\left(\xi, \xi_{0}\right) y^{\xi}\right|_{S} \rightarrow y^{\xi_{0}}\right|_{S} \quad \text { in } \quad l_{\varphi}^{2}(S)
$$

as $K \ni \xi \rightarrow \xi_{0}$. 
Here $l_{\varphi}^{2}(S)$ is the Hilbert space of all real-valued functions on $S$ with scalar product (24).

Proof. For any $\xi^{\prime}, \xi \in K$ set

$$
c\left(\xi^{\prime}, \xi\right):= \begin{cases}1, & \text { if }\left(y^{\xi^{\prime}}, y^{\xi}\right)_{\varphi} \geq 0 \\ -1, & \text { otherwise }\end{cases}
$$

The rest of the proof is similar to the proof of Lemma 5 .

Corollary. Let $\xi_{0} \in K$. There exists a strictly positive $\delta$ such that for any $\xi, \xi^{\prime} \in K$ with $\left|\xi-\xi_{0}\right| \leq \delta,\left|\xi^{\prime}-\xi_{0}\right| \leq \delta, \xi \neq \xi^{\prime}$, we have: $E \neq E^{\prime}$.

Proof. In the converse case, there exist two sequences $\xi_{j} \rightarrow \xi_{0}, \xi_{j}^{\prime} \rightarrow \xi_{0}\left(\xi_{j} \in K\right.$, $\xi_{\jmath}^{\prime} \in K, \xi_{j} \neq \xi_{j}^{\prime}$ ) such that $E_{\jmath}=E_{j}^{\prime}, j=1,2, \ldots$.. Lemma 10 shows that $\left(y^{\xi_{j}}, y^{\xi_{j}^{\prime}}\right)_{\varphi}=0, j=1,2, \ldots$, while by Lemma 11

$$
\left|\left(y^{\xi_{j}}, y^{\xi_{j}^{\prime}}\right)_{\varphi}\right| \rightarrow\left\|y^{\xi_{0}}\right\|_{\varphi}^{2}=1
$$

Proof of Proposition $4^{*}$ (the end). We have: $p(K)$ is nowhere dense but $\pi(K)$ contains some segment $I$. As above, the compact set $K$ may be thought of as minimal: if $K^{\prime} \subset K, K^{\prime} \neq K, K^{\prime}$ compact, then $\pi\left(K^{\prime}\right)$ does not contain the segment $I$.

Now make use of a sequence of functions on $K$ :

$$
J_{m}(\xi):=\sum_{n=-m}^{m}\left(y^{\xi}(n)\right)^{2}, \quad(\xi \in K, m=1,2, \ldots) ;
$$

they are continuous by Lemma 11 and converge pointwise to the function

$$
J_{K}(\xi)=\left\|y^{\xi}\right\|^{2}, \quad(\xi \in K) .
$$

We see as above that $J_{K}(\xi)$ has a point of continuity $\xi_{c} \in K$. Then we establish the following statement, analogous to Lemma 9.

Lemma 12. As $\xi, \xi^{\prime}$ tend to $\xi_{c}\left(\xi, \xi^{\prime} \in K ; \xi \neq \xi^{\prime}\right)$,

$$
\frac{t-t^{\prime}}{E-E^{\prime}} \rightarrow J_{K}\left(\xi_{c}\right)
$$

(Note that $E \neq E$ for small enough $\left|\xi-\xi_{c}\right|,\left|\xi^{\prime}-\xi_{c}\right|$, by Corollary to Lemma 11.)

The end of the proof, that is proving the fact that the compact set $K$ is not minimal (contrary to the hypothesis), is similar to the corresponding part of the proof of Theorem 1. This completes the proof of Proposition $4^{*}$ and thereby that of Theorem 2.

The proof of Theorem 5 is carried out along the same lines as that of Theorem 1 . The part of the solution $y^{\xi}$ to $(10)$ is played by the solution $y_{\vartheta, E}(\cdot)$ to the Cauchy problem

$$
\begin{gathered}
-y^{\prime \prime}+v(x) y=E y, \quad(x \geq 0), \\
y(0)=\sin \vartheta, \quad y^{\prime}(0)=\cos \vartheta
\end{gathered}
$$


(if it is square-integrable), and the part of Lemma 1 by the identity

$$
\sin \left(\vartheta_{1}-\vartheta_{2}\right)=-\left(E_{1}-E_{2}\right) \int_{0}^{\infty} y_{E_{1}, \vartheta_{1}}(x) y_{E_{2}, \vartheta_{2}}(x) d x
$$

Furthermore, instead of the spectral measure $\mu_{t}$, specified by (17), we use the measure $\varrho=\varrho^{\vartheta}$, defined in [CL], and instead of Lemma 8, the identity $\int_{0}^{\pi} \varrho^{\vartheta}(\Delta) d \vartheta=|\Delta|$ (see
$[\mathrm{Ko}]$ ).

Finally, the proof of Theorem 4 is derived from that of Theorem 5 in the same manner as the proof of Theorem 2 from that of Theorem 3.

\section{Proofs of "Random" Theorems}

Proof of Theorem $2^{*}$. Let $\mu_{j}(\omega)$ be the spectral measure of $e_{j}$ (the vector of the canonical basis, associated to site $j \in \mathbb{Z}$ ) for $h(\omega), L_{\jmath}^{\omega}$ be the $h(\omega)$-invariant subspace of $l^{2}(\mathbb{Z})$ generated by $e_{\jmath}$, and $\Sigma_{j}^{\omega}$ be the essential spectrum of $\left.h(\omega)\right|_{L_{j}^{\omega}}$. Obviously, $\Sigma_{j}^{\omega}$ consists of all non-isolated points of the support of the measure $\mu_{\jmath}(\omega)$. Fix $j=0$.

Lemma 13. $\Sigma_{0}^{\omega}=\Sigma$ with probability 1 .

Proof. Assume the converse. It is easy to see that then there exists a rational interval $I$ such that $I \cap \Sigma \neq \emptyset$ but with a positive probability $\bar{I} \cap \Sigma_{0}^{\omega}=\emptyset$. ( $\bar{I}$ denotes the closure of $I$.) The latter relation implies that the restriction of the spectral measure $\mu_{0}(\omega)$ to $\bar{I}$ consists only of a finite number of atoms: $\left|\sigma\left(\left.h(\omega)\right|_{L_{0}^{\omega}}\right) \cap \bar{I}\right|=: n_{0}(\omega)<\infty$. [Here $\sigma(B)$ denotes the spectrum of an operator $B$.) With the same positive probability we have for any $j \in \mathbb{Z}: \bar{I} \cap \Sigma_{j}^{\omega}=\emptyset$, or $\left|\sigma\left(\left.h(\omega)\right|_{L_{j}^{\omega}}\right) \cap \bar{I}\right|=: n_{j}(\omega)<\infty$.

These events, for some $j$ and $j^{\prime}\left(j<j^{\prime}\right)$, must combine, so that with a positive probability the spectra of restrictions of $h(\omega)$ to $L_{j}^{\omega}$ and $L_{j^{\prime}}^{\omega}$ are finite in $\bar{I}$ [consist of $n_{j}(\omega)$ and $n_{j^{\prime}}(\omega)$ points respectively].

It is easy to see that the deficiency index of the $h(\omega)$-invariant subspace of $l^{2}(\mathbb{Z})$, generated by $e_{j}$ and $e_{j^{\prime}}$, is not greater than $j^{\prime}-j-1$. Consequently, with a positive probability

$$
|\sigma(h(\omega)) \cap \bar{I}| \leq n_{\jmath}(\omega)+n_{j^{\prime}}(\omega)+\left(j^{\prime}-j-1\right)<\infty,
$$

so that

$$
\sigma_{\mathrm{ess}}(h(\omega)) \cap I=\emptyset
$$

Since with probability 1

$$
\sigma_{\mathrm{ess}}(h(\omega))=\Sigma,
$$

we obtain $\Sigma \cap I=\emptyset$, which contradicts the assumption. Lemma 13 is proved.

By the hypothesis, int $\Sigma \neq \emptyset$. Lemma 13 shows that for all $\omega$ in some set $\Omega_{0}$ of full measure in the probability space $\Omega$, we have $\Sigma_{0}^{\omega}=\Sigma$. Applying Theorem 1 to the restrictions $\left.h_{t}(\omega)\right|_{L_{0}^{\omega}}(t \in \mathbb{R})$, we obtain: if $\omega \in \Omega_{0}$, then $\sigma_{p}\left(\left.h_{t}(\omega)\right|_{L_{o}^{\omega}}\right) \cap \Sigma=\emptyset$ for all $t \in \mathbb{R} \backslash Z(\omega)$, where $Z(\omega)$ is thin. Since $\overline{\sigma_{p}(B)} \cup \sigma_{c}(B)=\sigma(B)$ for any selfadjoint operator $B$, we see that for the above $\omega, t$ the relation $\sigma_{c}\left(\left.h_{t}(\omega)\right|_{L_{0}^{\omega}}\right) \supset$ int $\Sigma$ holds. Theorem $2^{*}$ is proved. 
Proof of Theorem $2^{* *}$. By virtue of Theorem 2, it is enough to prove that a.s. $\sigma_{0}=\emptyset$ : no eigenfunction of $h(\omega)$ vanishes on $S$. Note that we impose no restrictions on the distribution $\nu$ of $v(0)$.

Without loss of generality, we may assume that $S=\{0\}$. Furthermore, it is enough to prove that for some fixed countable set $W \subset \mathbb{R}$, a.s. $\sigma_{0} \backslash W=\emptyset$ : since, according to [P], any fixed $E$ is an eigenvalue of $h(\omega)$ only with zero probability, then the proof will be complete. The countable set $W$ will be specified below.

In the notation of independent random coefficients $v(n, \omega)$ we will omit $\omega$. A real number $E$ belongs to $\sigma_{0}$ if and only if $E$ is an eigenvalue of each of the two random operators $h^{+}$and $h^{-}$, i.e. $\sigma_{0}=\sigma_{p}\left(h^{-}\right) \cap \sigma_{p}\left(h^{+}\right)$. Here $h^{+}$is a selfadjoint operator in $l^{2}(\mathbb{N})$ specified by the difference operation $l^{+}$:

$$
\left(l^{+} y\right)(n)=y(n-1)+y(n+1)+v(n) y(n) \quad(n \geq 1)
$$

and the boundary condition $y(0)=0 ; h^{-}$is defined similarly in $l^{2}(-\mathbb{N})$.

Since the restrictions of $v$ to $\mathbb{N}$ and to $-\mathbb{N}$ are independent of each other and the random set $\sigma_{p}\left(h^{-}\right)$is at most countable, the set $\sigma_{0} \backslash W \equiv\left(\sigma_{p}\left(h^{-}\right) \backslash W\right) \cap \sigma_{p}\left(h^{+}\right)$is a.s. empty if for any fixed $E \in \mathbb{R} \backslash W$ a.s. $E \notin \sigma_{p}\left(h^{+}\right)$.

Assume that for some fixed $E$ with a positive probability the solution $y(n)$ of the random Cauchy problem

$$
\begin{gathered}
\left(l^{+} y\right)(n)=\operatorname{Ey}(n) \quad(n \geq 1), \\
y(0)=0, \quad y(1)=1
\end{gathered}
$$

belongs to $l^{2}(\mathbb{N})$. The intersection of this event and its $j$-shift, for some $j>0$, has a positive probability (we use the shift invariance of the probabilistic measure). Since a square summable solution of (27), if any, is unique up to a constant multiplier, it follows that with a positive probability the solution $y(\cdot)$ to the above Cauchy problem vanishes at site $j$. Let $j$ be the minimal positive integer with this property. Therefore, the event

$$
X:=\{y(j)=0 ; y(n) \neq 0(n=1,2, \ldots, j-1)\}
$$

has a positive probability.

Consider an at most countable set $N:=\{E \in \mathbb{R} \mid \nu(\{E\})>0\}$ of all atoms of the distribution $\nu$ and define events $Y, Y_{1}, Y_{2}, \ldots, Y_{\jmath-1}$, whose union is the whole probability space $\Omega$ :

$$
\begin{gathered}
Y:=\{v(n) \in N(n=1,2, \ldots, j-1)\} ; \\
Y_{k}:=\{v(k) \notin N\}, \quad(k=1,2, \ldots, j-1) .
\end{gathered}
$$

First,

$$
\operatorname{Pr}\left(X \cap Y_{k}\right)=0, \quad(k=1,2, \ldots, j-1) .
$$

Indeed, fixing all $v(n)(n \neq k)$ and varying $v(k)$, find the conditional probability of the event $X \cap Y_{k}$. Since $y(k) \neq 0$ (by the definition of $X$ ), there is only one value of $v(k)$ for which $y(j)=0$. (Proof: if $v^{*}(k)$ is another value with the same property, then, by Green's formula, $\left.\left(v^{*}(k)-v(k)\right) \cdot(y(k))^{2}=0\right)$. As $v(k) \notin N$, the above conditional probability is zero, and hence $(28)$ is true.

Furthermore, let the event $X \cap Y$ take place. Note that $y(j)$ is a polynomial in $E$ of degree $j-1$, whose coefficients depend on $v(1), v(2), \ldots, v(j-1)$. Each of them belongs to the countable set $N$, and hence $E$ must belong to the countable set $\mathscr{Z}$ of zeros of all the corrresponding polynomials. If we set $W:=\mathscr{Z}$, then for $E \in \mathbb{R} \backslash W$ 
the event $X \cap Y$ is impossible. In view of (28), we have $\operatorname{Pr}(X)=0$. Theorem $2^{* *}$ is proved.

Acknowledgements. It is a pleasure to thank S. A. Molchanov for his encouragement to find out whether or not the exceptional $\theta$ 's exist in the Kotani theorem and in the example [Go]. I am also grateful to Ya. G. Sinai and E. I. Dinaburg for their attention to this work and fruitful discussions of its results.

Note added in proof. Some results close to ours were obtained independently by R. del Rio, N, Makarov, B. Simon (see [RJMS], [S]). Their method of proof is different from outs.

\section{References}

[AG] Akhiezer, N.I., Glasman, I.M.: Theory of linear operators in Hilbert space (in 2 volumes), vol. 2. New York: Ungar Publ., 1963

[CL] Coddington, E.A., Levinson, N.: Theory of ordinary differential equations. New York: McGraw-Hill 1955

[CD] Chulayevsky, V.A., Sinai, Ya.G.: Anderson localization for the 1-D discrete Schrödinger operator with two-frequency potential. Commun. Math. Phys. 125, 91-112 (1989)

[DLS] Delyon, F., Lévy, Y., Souillard, B.: Anderson localization for one- and quasi-one-dimensional systems. J. Stat. Phys. 41, No. 3/4, 375-388 (1985)

[DS] Dunford, N., Schwarz, J.: Linear operators. Spectral theory

[Gl] Glazman, I.M.: Direct methods of qualitative spectral analysis of singular differential operators. Moscow: Nauka 1963 (in Russian)

[Go] Gordon, A.Ya.: Determinate potential with pure point spectrum. Mat. Zametki 48, No. 6, 38-46 (1990) (in Russian)

$\left[\mathrm{Go}^{\prime}\right]$ Gordon, A.Ya.: On exceptional values of the boundary phase for the Schrödinger equation on a half axis. Russian Math. Surveys. 47, 211-212 (1992) (in Russian)

[KMP] Kirsch, W., Molchanov, S.A., Pastur, L.A.: One-dimensional Schrödinger operator with an unbounded potential: Pure point spectrum. Funkt. Anal. i Prilozhen. 24, No. 3 (1990) (in Russian)

[Ko] Kotani, S.: Lyapunov exponents and spectra for one-dimensional Schrödinger operators. AMS Series of Contemp. Math. 50, 277-286 (1986)

[O] Oxtoby, J.C.: Measure and category. Berlin, Heidelberg, New York: Springer 1971

[P] Pastur, L.: Spectral properties of disordered systems in one-body approximation. Commun. Math. Phys. 75, 179 (1980)

[RJMS] Del Rio, R., Jitomirskaya. S., Makarov, N., Simon, B.: Singular continuous spectrum is generic (preprint)

[S] Simon, B.: Spectral analysis of rank one perturbations and applications. Lecture notes. Vancouver, 1993

[SW] Simon, B., Wolff, T.: Singular continuous spectrum under rank one perturbations and localization for random Hamiltonians. Commun. Pure Appl. Math. 39, 75-90 (1986) 
